\title{
Development of an Intelligent Virtual Grader for Estimation of Fruit Quality
}

\author{
Ajay Pal Singh Chauhan \\ Department of ECE, \\ Sant Longowal Institute of Engg.\& Tech., \\ Longowal Distt. Sangrur, Punjab (INDIA)
}

\author{
Amar Partap Singh \\ Department of ECE, \\ Sant Longowal Institute of Engg. \& Tech., \\ Longowal Distt. Sangrur, Punjab (INDIA)
}

\begin{abstract}
Food industry is amongst the industries that largely use machine vision for inspection of produce. Machine vision systems benefit from specially designed digital image processing software to accomplish this task. Color provides valuable information in examining the freshness and estimating the maturity of fruits. The development of an intelligent virtual grader is presented for automatic grading of red delicious apple fruits based on their surface color using machine vision. The heart of the proposed virtual grader is executed in the form of k-nearest neighbor classifier. k-nearest neighbor classifier is chosen for this particular application since it is more robust to noise as compared to other classification algorithms.The performance of the implemented virtual grader is examined experimentally with an industrial grade camera connected to an image grabber of a computer based machine vision system. Results of this study are quite promising. In fact, efficiency achieved using proposed virtual grader is $95.12 \%$ if manual grading taken as reference level is assumed to be $100 \%$ accurate. However, after having rigorous experimental validation the repeatability of the proposed system is found to be $100 \%$.
\end{abstract}

\section{Keywords}

Intelligent, virtual, grader, fruit, color, classifier and quality

\section{INTRODUCTION}

Machine vision proved to be an effective tool that could be used to replace human sorters for reliable and consistent judgment in estimating and comparing color of the fruits as human inspectors makes different judgments on the same product at different instances. So we can affirm that the only technology that can accomplish this task is machine vision. In classification an unknown sample is identified by comparing a set of its significant features to a set of features that represent classes of known samples [1-4]. Fruits are graded based on their external factors like shape, size, color and external damage, etc. Color plays a vital role in the classification of the fruits. Classification of fruits based on color is the most important tasks performed in the packaging industry. Various color classification algorithms are reported for the purpose of object recognition. Accordingly, a number of solutions are proposed for identifying different types of fruits. However, color classification algorithms employing the concept of machine learning have been used rarely for the purpose of assessing the quality of a particular type of fruit. Based on these considerations, an attempt is made to extend the applicability of color classification for the purpose of assessing the quality of a particular type of fruit using machine vision[5][6]. In order to do so, there is an urgent need to introduce artificial intelligence in machine vision thus making it fit for exercising an effective judgment for inspection of produce similar to human quality inspectors. Different color analysis methods are available to evaluate the quality of fruit on the basis of surface color. These methods include neural networks, statistical analysis and mathematical analysis. In the process of color classification, extraction of useful information concerning the spectral properties of object by discovering the best match from a set of known descriptors or class models to implement the recognition task is involved. Database is also available for manual inspection of apple fruit. Visual color classification system was reported using RGB color model histograms in which mean value and color quantization value represents the scanned color images [7]. FPGA based system was also designed using color space in which RGB was first converted into the YIQ [8]. Here, RGB color space was separated into a luminance part and two chrominance parts. Then, linear transform was used to convert the RGB values into YIQ easily. Study on HSI color model based fruit quality evaluation was also reported [9]. In this study, the RGB model was first converted into HSI (or HSL) model and simplified histogram of hue was calculated, which was designed using BP Neural Network [10]. It has been found experimentally that in finding the ripeness of Apple, HSI (or HSL) model is most suitable. In another work, after performing normalization, RGB values were first converted into HSI using a set of converting equations in $\mathrm{C}++$. Statistical Analysis is useful only when we use RGB. In this classification, based on hue histogram distribution, median density of hue of the apple was used as the basis for grading [11].Neural Network and Nearest Neighbor was used to evaluate the quality of an image and recognition of stem and calyx in a Window Based Method for Automatic Classification of Apple Fruit [12]. In another work fruit sorting was realized by classic Bayes classifier, whose parameter were obtained by a study module and in this crystal Fuji apples were used to test the system with average sorting accuracy of $90 \%$ [13].Proposed virtual grader is designed using HSL color space. However, in the proposed work, all the three components of the HSL color space, that is hue, saturation and luminance constitute the color features that forms the basis for the classifier to make distinction between different grades of the fruit. An attempt is made to develop a new type of virtual grader to estimate the apple quality from its surface color.

\section{K-NEAREST NEIGHBOR CLASSIFIER}

Color classifier uses color features to identify samples based on their color. Classification involves two phases: 
training and classifying. Training is a phase during which the machine vision software is taught, the types of samples

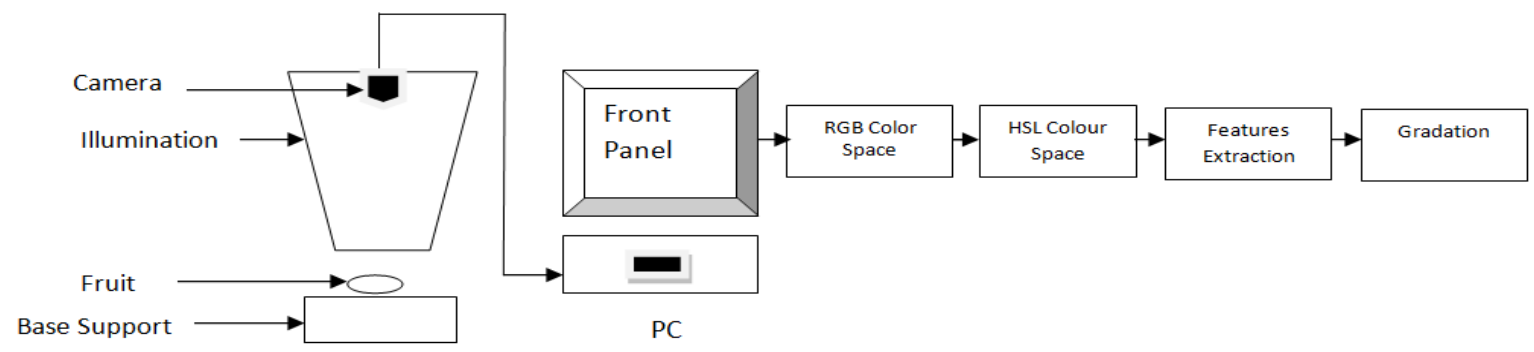

Fig. 1: Experimental set up

need to be classified during the classifying phase. Any number of samples can be trained to create a set of classes, which are used on later stage to compare unknown samples during the classifying phase. Classes are stored in a classifier file. Training might be a one-time process, or it might be an incremental process that repeats to add new samples to existing classes or to create several classes, thus broadening the scope of samples required to be classified. Classifying is a phase during which the custom machine vision application classifies an unknown sample in an inspection image into one of the classes that are trained. The classifying phase classifies a sample according to how similar the sample features match features of the trained samples [2]. Though various color classification algorithms such as k-Nearest Neighbor, Nearest Neighbor and Minimum Mean distance can be used but K nearest Neighbor is preferred as it is best suited for this particular application since it is more immune to noise compared with other classification algorithms.

The k-Nearest Algorithm is a method for classifying objects based on closest training examples in the color feature space. $\mathrm{k}-\mathrm{NN}$ is a type of instance based learning where the function is only approximated locally and all computation is deferred until classification. Amongst all machine learning algorithm k-nearest neighbor algorithm is the simplest. An object is classified by majority vote of its neighbors with the object being classified to the class most common amongst its $\mathrm{k}$ nearest neighbors. If $\mathrm{k}$ is equal to one, then the object is simply assigned to the class of its nearest neighbor. In k-nearest neighbor classification, a color is classified into a class based on a voting mechanism. National Instruments Classifier is used for color classification.

\section{DEVELOPMENT OF PROPOSED INTELLIGENT VIRTUAL GRADER}

Development of the proposed intelligent virtual grader involves following steps:

\subsection{Preparation of Training Samples}

Two hundred apples of red delicious variety were taken covering a wide range of its quality. Three human experts trained in the field of manual grading of this particular variety of apples were given the chosen samples. The human experts graded the apples as per CODEX Stan 299 Standard for apples which says that apples must have some definite value of red color content. So four grades A, $\mathrm{B}, \mathrm{C}$ and D having 25 samples each was selected based on red color content. Fifteen samples in each grade were taken as reference samples that were commonly agreed upon by all the experts. NI Vision Builder for Automated Inspection software is used to acquire images using experimental set up shown in Fig 1. In the proposed experimental setup, image acquisition card, industrial grade BASLER sca-1390 17fc color camera, NI Vision builder and LabVIEW software are executed on a computer. The image taken is digitized as 24-bit RGB data at the resolution of $1390 \times 1038$. Data is then processed and analyzed using color classification interface available in NI Vision Builder. Color Classification Training Interface is used to train a color classifier by manually classifying color samples into different color classes.

\subsection{Implementation of Image Processing Software}

The image processing software, the heart of the proposed virtual grader, is executed in the form of color classifier using NI Vision Builder for Automated Inspection. NI Vision Builder is chosen for the software development in LabVIEW environment, as it provides an effective graphical user interface. K-nearest neighbor algorithm is used to execute the color classifier designed around Euclidean metrics. The executed color classifier employs HSL color space to calculate a color feature for every sample to be trained or classified. The color classifier calculates the color features in which the color sample is first converted from RGB to HSL color space. Then hue, saturation and luminance histograms of the color sample are computed. The hue and saturation histograms each contain 256 values. Luminance histogram is reduced to 8 values which are suppressed by $12.5 \%$. By suppressing the luminance histogram, the executed color classifier accentuates the color information for the sample. In the end, 520 values of hue, saturation and luminance are combined to produce a high resolution color feature. Further, medium resolution color features are obtained by applying a dynamic mask to the high resolution color feature. The medium color features are subsets of high resolution color feature. The medium resolution color feature contains 128 hue and saturation values and 8 luminance values for a total of 136 values. The medium resolution feature is used in the proposed work to speed up color classification process. The diagram shown in Fig. 2 displays the back end programming of the system. In fact, 
it decimates the main blocks into sub blocks like preprocessing, processing, post processing and classification.
The inspection image contains multiple samples. These were fed to the classifier for training. The region of interest

\subsection{Training of Color Classifier}

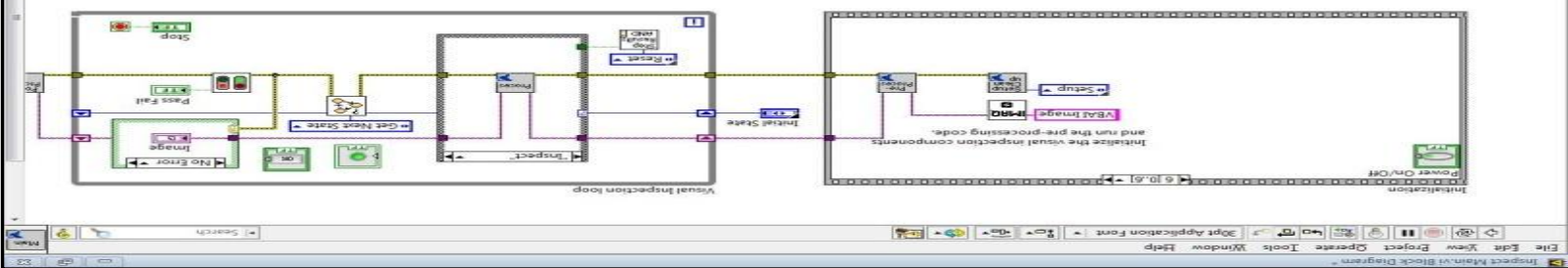

Fig.2 Back end Programming of the proposed Intelligent Virtual Grader

toolbar is used to define a region which is useful for training. The Annulus ROI tool is chosen for apple because it adjusts the inner and outer radii, and also adjusts the start and end angles. The executed color classifier has two phases that is the training phase and a classifying phase. In the training phase, the classifier is provided with 15 samples in each grade, i.e., A, B, C and D. A known sample consists of a region in the image, containing the color the classifier needs to learn. The color classifier calculates a color feature and assigns the associated class label to the feature, for each and every sample added during the training phase. Color feature represents three dimensional color information of the sample in a one dimensional format. Eventually, all the trained samples added to the classifier are saved into a file which represents a trained color classifier. After training the classifier, regions are classified into their corresponding classes for color identification in the apple quality. Experimentation has been conducted using database of 60 training samples as selected by three human experts, 15 samples in each category. The optimal value of number of training samples $(\mathrm{K})$ and illumination intensity are determined after rigorous experimental evaluation of the executed color classifier. After having rigorous experimental trial, it is found that the proposed classifier works effectively at an illumination intensity of 310 lux when number of training samples taken is 11 . Using these training samples, the engine method namely k-Nearest

Neighbor algorithm with Euclidean metrics was trained. In the testing phase, the selected 60 test samples as selected by human experts are given to the proposed machine vision system which classifies and grade them according to their color content. In this classifying phase, the classification engine calculates the color features of the sample that need to be identified and classify it among trained sample using k-Nearest Neighbor Algorithm. The Classification process is responsible to classify the input or user selected fruit by using k-NN algorithm. This measure the distance between features values of the stored fruit test. Afterward the k-NN finds out among the stored fruit example that have shortest distance with the input. Later than the system will identify and assign the class to the input fruit.

\subsection{Synthesis of Front Panel}

Front Panel is designed to serve the purpose of user interactive interface where actual testing of apple will take place. The Front panel is used to acquire the apple image automatically under the supervision of a high performance algorithm of control and computations. After acquisition of the image, the Front Panel displays corresponding grade and other related parameters as estimated by the Signal Processing Software. The user-interactive Front Panel is designed in a fourth generation, graphical integrated programming language using LabVIEW software and is highly customized for this particular application. The different LabVIEW objects were carefully selected, researched, configured and interlinked to develop an elegant user-interactive Front-panel, as shown in Fig 3. The K-Nearest Neighbor Based Color Classifier executed in NI Vision Builder is coded into LabVIEW environment to integrate it into the Front Panel. The source code for the successful action of the Front Panel was written as a complete integrated module in the form of a Block diagram as shown in Fig.2 to perform various actions.

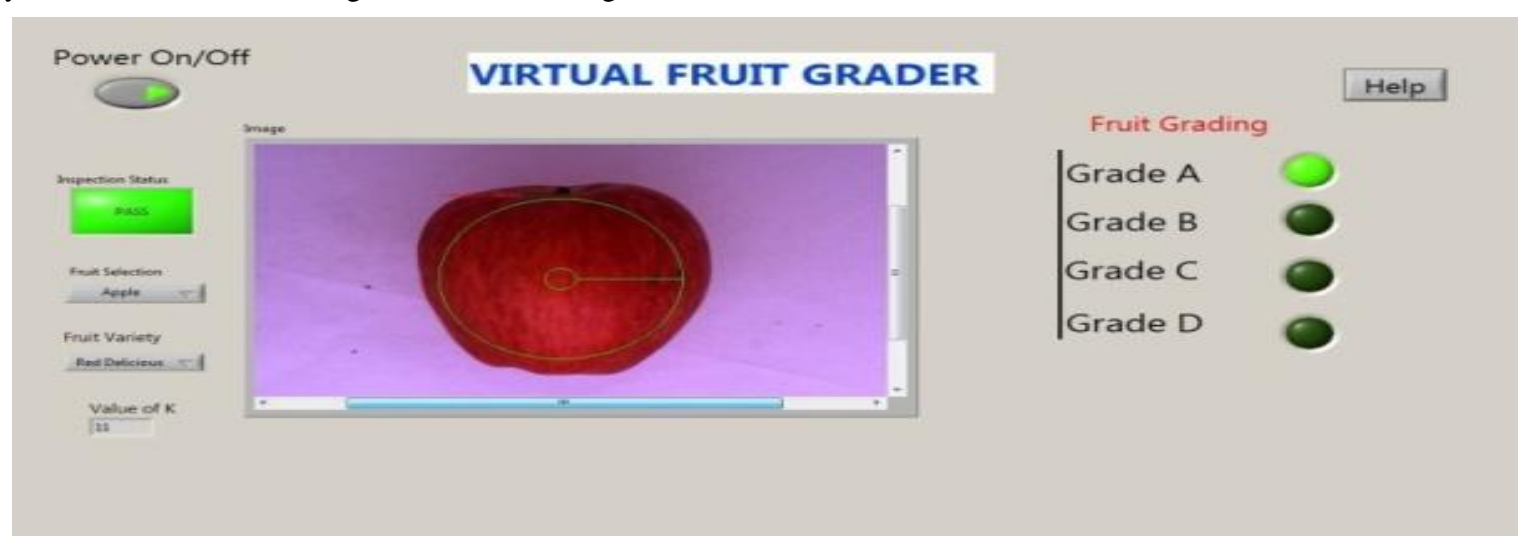

Fig.3: Front panel of the proposed Intelligent Virtual Grader 


\subsection{Operation of the Proposed Intelligent Virtual Grader}

The operation of the proposed intelligent virtual grader is controlled by a high performance algorithm of control and computations as explained in the earlier section. The algorithm of control and computations coordinate the functioning of various modules of the proposed intelligent virtual grader. The virtual grader is designed to operate in a user interactive manner. However, in order to study the effective use of proposed virtual grader, it is operated practically in real time environment. Accordingly, the performance of the virtual grader is found to be quite satisfactorily as confirmed from the results presented in the following section.

\section{RESULTS AND DISCUSSION}

The implemented intelligent virtual grader is used practically to estimate the quality of red delicious apple fruits as shown in Fig.2. In order to validate the effectiveness of the proposed intelligent virtual grader, it is brought in the operational phase by switching it on. In operational phase, the color classifier operates in the classifying phase and the virtual grader acquires the images automatically. As soon as the image of the apple under test is acquired, it automatically displays its grade along with other related parameters in the respective objects configured on the Front Panel. In fact, efficiency achieved using proposed classifier is $95.12 \%$ if manual grading taken as reference level is assumed to be $100 \%$ accurate. However, $4.88 \%$ variation is also due to subjective judgment of human-beings in perceiving the apple fruit during manual grading, which of course is inevitable. Moreover, the repeatability of the proposed system is found to be $100 \%$ from rigorous experimental validation. However, the manual grading is always doubted due to subjective tolerance. This fact is also confirmed by three experts chosen for manual grading. According to them, it is not possible for them to decide the border cases. However, to confirm the effectiveness of the proposed system, it is found through rigorous experimentation that the repeatability of the proposed virtual grader is found to be $100 \%$. Table- 1 shows the range of values of average and standard Deviation of the training samples for a particular grade. These are required to train the classification engine. Color grading using RGB color space is complicated by the 3D representation of colors thus making the selection and adjustment of colors difficult. From the result of present study, it has been verified experimentally that HSL color model is comparatively more effective as compared to RGB model for gradation of same type of fruit as well as color classification. The range of HSL values of average and standard deviation are listed in Table- 2 . The proposed color classifier first converts RGB color space to HSL model. As this algorithm classifies color based on hue and saturation only, so it will work efficiently accordingly to the range of values for different grades.

\section{CONCLUSION}

The implemented system can be used effectively to grade red delicious apple based on their color features. It has been found experimentally that k-Nearest Neighbor Classifier employing Euclidean Distance Metric achieved promising results for this particular application. It is found that efficiency is the highest at a particular value of illumination intensity as well as number of training samples. After analyzing the percentage accuracy achieved with the proposed method, it is concluded that k-Nearest Neighbor algorithm with Euclidean metric is an efficient algorithm to translate effectively human visual perception about grading the apple in to machine vision. The virtual grader predicts the right quality of apple successfully when checked for complete set of apples used for training as well as in classifying phases. The results obtained in the real time operation of the proposed intelligent virtual grader confirms the results obtained in the training and classifying phases. The theoretical principles and practical design of the proposed virtual grader are described. The technique would be quite useful for other types of fruits possessing similar surface characteristics. In fact the proposed technique has a potential future in the field of machine vision based inspection of agriculture produce.

\section{ACKNOWLEDGMENTS}

The authors like to thank Department of ECE SLIET Longowal for providing the lab facility as without that it would not have been possible to complete this work.

Table1. Range of RGB values of the training samples

\begin{tabular}{|c|c|c|c|c|c|c|}
\hline Grade & Red Av. & Red Std. Dev & Green Av. & Green Std Dev & Blue Av. & Blue Std. Dev. \\
\hline A & $100-134$ & $26-40$ & $7.2-17$ & $8-15.3$ & $12-18$ & $10-14$ \\
\hline B & $138-172$ & $32-48$ & $17.4-30$ & $15.3-20$ & $20-32$ & $14-20$ \\
\hline C & $137-170$ & $33-53$ & $21.2-43.9$ & $12.7-33.7$ & $24-38$ & $14-26$ \\
\hline D & $175-220$ & $34-58$ & $65-102$ & $32.9-54.9$ & $46-74$ & $24-42$ \\
\hline
\end{tabular}

Table2. Range of HSL values of the training samples

\begin{tabular}{|c|c|c|c|c|c|c|}
\hline Grade & Hue Av. & Hue Std. Dev & Sat. Av. & Sat. Std Dev & Lum Av. & Lum. Std. Dev. \\
\hline A & $254-255$ & $0.8-1.4$ & $198-216$ & $29-39$ & $33-53$ & $13-22$ \\
\hline B & $253-254$ & $1.3-1.8$ & $177-198$ & $28-30$ & $52-79$ & $21-28$ \\
\hline C & $250-253$ & $1.6-2.2$ & $166-178$ & $26-28$ & $80-90$ & $20-22$ \\
\hline D & $241-250$ & $2.8-6.1$ & $113-138$ & $20-26$ & $100-130$ & $33-47$ \\
\hline
\end{tabular}




\section{REFERENCES}

[1] http://zone.ni.com/reference/enXX/help/372916H01/niv isionconcepts/classification_methods/

[2] NI Vision Concepts Manual, August 2009

[3] NI Vision Builder for Automated Inspection Manual, August 2009.

[4] Jeffrey Travis and Jim Kring, "LabVIEW for Everyone Graphical Programming made easy and Fun" $\left(2009^{\text {th }}\right.$ Edition), Pearson Education, 41-43

[5] Rafael C. Gonzalez and Richard E. Woods, "Digital Image Processing", ( $3^{\text {rd }}$ Edition), Pearson Education, 488-499

[6] Anil K Jain, "Fundamentals of Digital Image Processing", ( $3^{\text {rd }}$ Edition), Prentice Hall Edition, 132-134

[7] Ling Mei Chan, Rodney Tan and Gilbert Thio, "Design of Visual Based Color Classification System", JASA 2 (January 2007), 30-33.

[8] Balkrishan Ahirwal, Mahesh Khadtare and Rakesh Mehta, "FPGA based system for color space Transformation RGB to YIQ and YCbCr,"International Conference on Intelligent and Advanced systems (2007), 1345-1349.
[9] Yizhong Wang, Yanhua Chi, Huafang Huang, Shahui Chen, Ping Chang, George Q. Huang, "Study on HIS Color Model Based Fruit Quality Evaluation",

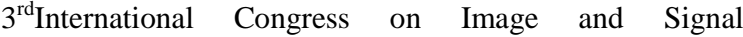
Processing(CISP-2010) vol 6, 2677-2680.

[10] Young-Chang and John F. Reid, "RGB Calibration for Color Image Analysis in Machine Vision", IEEE Transactions on Image Processing,Vol 5, No. 10, (October 1996), 1414-1422.

[11] P.Sudhakara Rao, A.Gopal, R. Revathy and K. Meenakshi "Colour Analysis of Fruits Using Machine Vision System for Automatic Sorting And Grading",J.Instrum.Soc.India 34(4), 284-291.

[12] Shivleela R Arlimatti , "Window Based Method for Automatic Classification of Apple Fruit" International Journal of Engineering Research and Applications Vol. 2, Issue 4, July-August 2012, 1010-1013.

[13] Feng G. and Qixin C., "Study on Color Image Processing Based Intelligent Fruit Sorting System", Proceedings of the 5th World Congress on Intelligent Control and Automation, Hangzhou, P.R. China.Vol. 6 June 15-19, $2004,4802-4805$ 